\title{
Second-line-Therapie mit Cabazitaxel
}

Das neue Taxan Cabazitaxel ist - anders als klassische Taxane wie beispielsweise Docetaxel - ein schlechtes Substrat für ABC-Transporter. Diese werden mit einer Taxan-Resistenz in Verbindung gebracht. Nach Axel Heidenreich, Aachen, ist Cabazitaxel für die Therapie von Patienten mitkastrationsresistentem Prostatakarzinom nach Docetaxel-Versagen vielversprechend.

Eine Chemotherapie mit Docetaxel kann laut der aktuellen S3Leitlinie zur Früherkennung, Diagnose und Therapie des Prostatakarzinoms bei Patienten mit symptomatischer progredienter Erkrankung unter medikamentöser Kastration und im guten Allgemeinzustand durchgeführt werden.

\section{Verlängertes Gesamtüberleben in Phase-III-Studie}

Phase-III-Daten zur Second-line-Therapie mit Cabazitaxel wurden kürzlich beim Genitourinary Cancers Symposium in San Francisco/ USA vorgestellt (Sartor AO et al., 2010, GU-ASCO: \#9, oral presentation). In der Studie TROPIC wurde mit Cabazitaxel im Vergleich zu Mitoxantron bei Patienten mit metastasiertem kastrationsresistentem Prostatakarzinom nach Docetaxel-Versagen eine Verlängerung des medianen Gesamtüberlebens erreicht (primärer Endpunkt; Abb.). In dieser Studie wurden 755 Patienten randomisiert mit Cabazitaxel

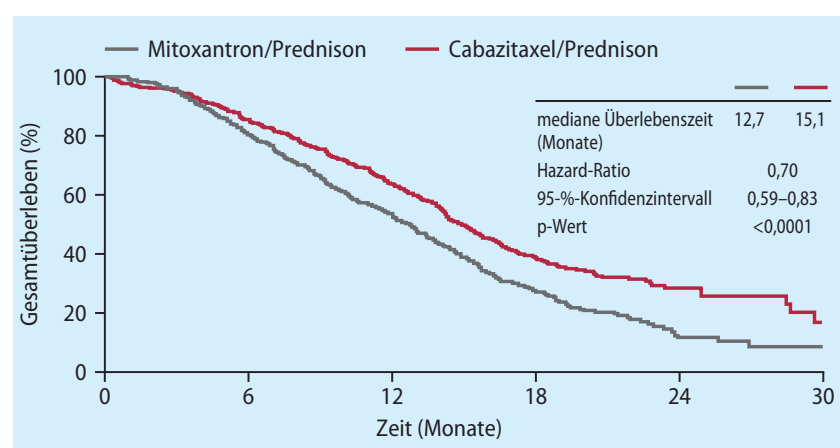

nach Sartor AO et al., 2010, ASCO-GU: \#9, oral presentation

TROPIC-Studie - Gesamtüberleben von Patienten mit metastasiertem kastrationsresistentem Prostatakarzinom nach Docetaxel-Versagen: Cabazitaxel versus Mitoxantron

oder Mitoxantron behandelt. Mitoxantron ist in dieser Situation das bisher einzige etablierte Zytostatikum neben Docetaxel. Vorteile zugunsten der Therapie mit Cabazitaxel zeigten sich auch bei sekundären Endpunkten wie beispielsweise dem progressionsfreien Überleben und der Tumoransprechrate. UH

Quelle: Veranstaltung und Informationen der Sanofi-Aventis Deutschland GmbH

\section{Ältere Tumorpatienten}

\section{Paradigmenwechsel durch neue Studiendaten}

Die Ansicht, eine Tumorbehandlung nutze jüngeren Patienten mehr als betagteren, lässt sich wissenschaftlich, insbesondere für neue innovative Behandlungskonzepte, nicht mehr aufrecht erhalten. „Das biologische und nicht das kalendarische Alter bestimmt die Therapie“, konstatierte Arnd Hönig, Würzburg.

Prognosen zu absoluten Häufigkeiten von Tumorerkrankungen gehen davon aus, dass bis zum Jahr 2020 die Zahl der jährlichen Neuerkrankungen bei Frauen, die älter als 65 Jahre sind, um 25\% steigen wird (Gesundheit in Deutschland. Robert Koch-Institut, Berlin 2007, S. 42). Ein Alter von mehr als 65 Jahren war früher in klinischen Studien ein Ausschlusskriterium für die Teilnahme. Demzufolge waren für diese Patientengruppe Aussagen über Wirksamkeit und Verträglichkeit vieler Medikamente nicht möglich.

\section{IN-GHO ${ }^{\circledR}$-Register und Senora}

DieWissenslücke wird zunehmend geschlossen, sagte Friedemann Honecker, Hamburg. Denn einerseits werden ältere Patienten in klinische Studien eingeschlossen und andererseits werden Daten aus Registerstudien gewonnen. So werden im IN-GHO ${ }^{\circledR}$-Register bis Ende 2010 Daten aus etwa 100 Zentren von mehr als 3.000 Tumorpatienten gesammelt, die mindestens 70 Jahre alt sind. Eine Zwischenauswertung von 1.580 Patienten zeigte keinen Einfluss des Patientenalters auf das Therapieergebnis. Das Mammakarzinom-Register Senora soll Daten von 1.000 Patientinnen einschließen, die älter als 70 Jahre sind.

\section{Mammakarzinom}

Die Phase-III-Studie AVADO verglich bei Patientinnen mit HER2-negativem metastasiertem Mammakarzinom die First-lineTherapie mit Docetaxel mit der Kombi- nationstherapie Bevacizumab (Avastin ${ }^{\circledast}$ ) plus Docetaxel. Wie die Ergebnisse der aktuellen Subgruppenanalyse zeigen, ist die Kombinationstherapie bei älteren Patientinnen genauso wirksam und verträglich wie bei jüngeren Patientinnen (Pivolt $X$ et al., 2009, J Clin Oncol 27: 15s, \#1094).

Auch für Patientinnen mit HER2-positivem Mammakarzinom zeigten die Ergebisse einer großen Beobachtungsstudie zum Einsatz von Trastuzumab (Herceptin ${ }^{\oplus}$ ) einen Vorteil für ältere Patientinnen: Das progressionsfreie Überleben der Patientinnen, die mindestens 65 Jahre alt waren, war im Vergleich zu jüngeren Patientinnen verlängert (median 12,9 Monate vs. 9,9 Monate; Jackisch C et al., 2008, SABCS: \#3144). FH

Quelle: Veranstaltung der Roche Pharma AG 